05. $1 ; 06.5 ; 10$

\title{
Влияние механических напряжений на поведение лазерных ультразвуковых сигналов вблизи отверстия в керамике нитрида кремния
}

\author{
(С) А.Л. Глазов, К.Л. Муратиков \\ Физико-технический институт им. А.Ф. Иоффре РАН, Санкт-Петербург, Россия \\ E-mail: glazov.holo@mail.ioffe.ru
}

Поступило в Редакцию 16 февраля 2021 г.

В окончательной редакции 26 марта 2021 г.

Принято к публикации 26 марта 2021 г.

Выполнено экспериментальное исследование поведения лазерных ультразвуковых сигналов вблизи отверстия в диэлектрической керамике нитрида кремния при воздействии внешних сжимающих напряжений. Показано принципиальное расхождение полученных результатов с аналогичными результатами, полученными нами ранее для металлов, и их количественное несоответствие предсказаниям термодинамической модели формирования термоупругих сигналов от напряженных материалов. Предложено объяснение наблюдающихся расхождений с помощью модели теплового возбуждения дефектных состояний решетки, предложенной нами ранее.

Ключевые слова: керамика, лазерный ультразвук, механические напряжения, термоупругость.

DOI: 10.21883/PJTF.2021.12.51067.18740

Лазерное возбуждение ультразвуковых колебаний в твердых телах широко используется при определении упругих и теплофизических свойств объемных материалов и тонких пленок $[1,2]$ для неразрушающего контроля изделий различного рода, включая изготовленные методами аддитивных технологий и 3D-печати [3]. Одним из основных механизмов лазерного возбуждения ультразвука является термоупругое взаимодействие. Считается, что к настоящему времени его основные характеристики достаточно хорошо проработаны. Вместе с тем в последнее время для проводящих объектов в ненапряженном состоянии были получены результаты, которые не согласуются с предсказаниями классической термоупругой модели [4-6]. Исследования лазерного возбуждения ультразвуковых колебаний в напряженных металлических объектах также позволили обнаружить существенное расхождение между экспериментальными результатами и предсказываемыми термодинамической моделью [7,8].

Основной целью настоящей работы является детальное исследование корректности описания процессов лазерной генерации в напряженных непроводящих керамиках с помощью современной термоупругой модели. Ранее нами были выполнены исследования подобного рода на керамике нитрида кремния [9] и композитной керамике $\mathrm{Al}_{2} \mathrm{O}_{3}-\mathrm{SiC}-\mathrm{TiC}[10,11]$. В этих работах изучалось поведение лазерных ультразвуковых сигналов вблизи концов приповерхностных трещин, образующихся при индентировании по Виккерсу. В них было показано, что поведение лазерных ультразвуковых сигналов в указанных областях качественно соответствует описанию в рамках стандартной термоупругой модели. Однако из-за сложной геометрии системы трещин детальное количе- ственное сравнение экспериментальных и теоретических результатов было сильно затруднено.

В настоящей работе исследовано поведение лазерных ультразвуковых сигналов в керамике вблизи неоднородности с хорошо заданной геометрией. В качестве такой неоднородности было выбрано отверстие, для которого существует точное аналитическое решение для распределения напряжений при воздействии на образец внешнего напряжения (задача Кирша [12]). В качестве материала образца была выбрана керамика нитрида кремния, у которой отсутствует электрическая проводимость. Эксперименты проводились на образце керамики нитрида кремния в виде параллелепипеда размером $12.5 \times 8.3 \times 2.4 \mathrm{~mm}$. В центре большой грани было сделано несквозное отверстие с помощью $\mathrm{CO}_{2}$-лазера. Диаметр отверстия на поверхности равнялся $0.6 \mathrm{~mm}$, а его глубина составляла $0.5 \mathrm{~mm}$. Керамика была изготовлена методом горячего прессования в направлении, перпендикулярном грани, на которой было проделано отверстие.

Оптоакустические изображения области вокруг отверстия были получены при двумерном сканировании лазерным лучом по поверхности с шагом $20 \mu \mathrm{m}$. Измерялся сигнал с цилиндрического пьезокерамического датчика, поджатого к тыльной стороне образца через тонкий слой гелеобразной акустической смазки, на частоте $101 \mathrm{kHz}$, близкой к резонансной частоте пьезодатчика. Результаты эксперимента представлены на рисунке. На рисунке, $a$ показана область размером $2 \times 2 \mathrm{~mm}$ в исходном ненагруженном образце. Распределение сигнала практически равномерное. На изображении одноосно нагруженного образца (см. рисунок, $b$ ) области с увеличенным и уменьшенным сигналом расположены диа- 

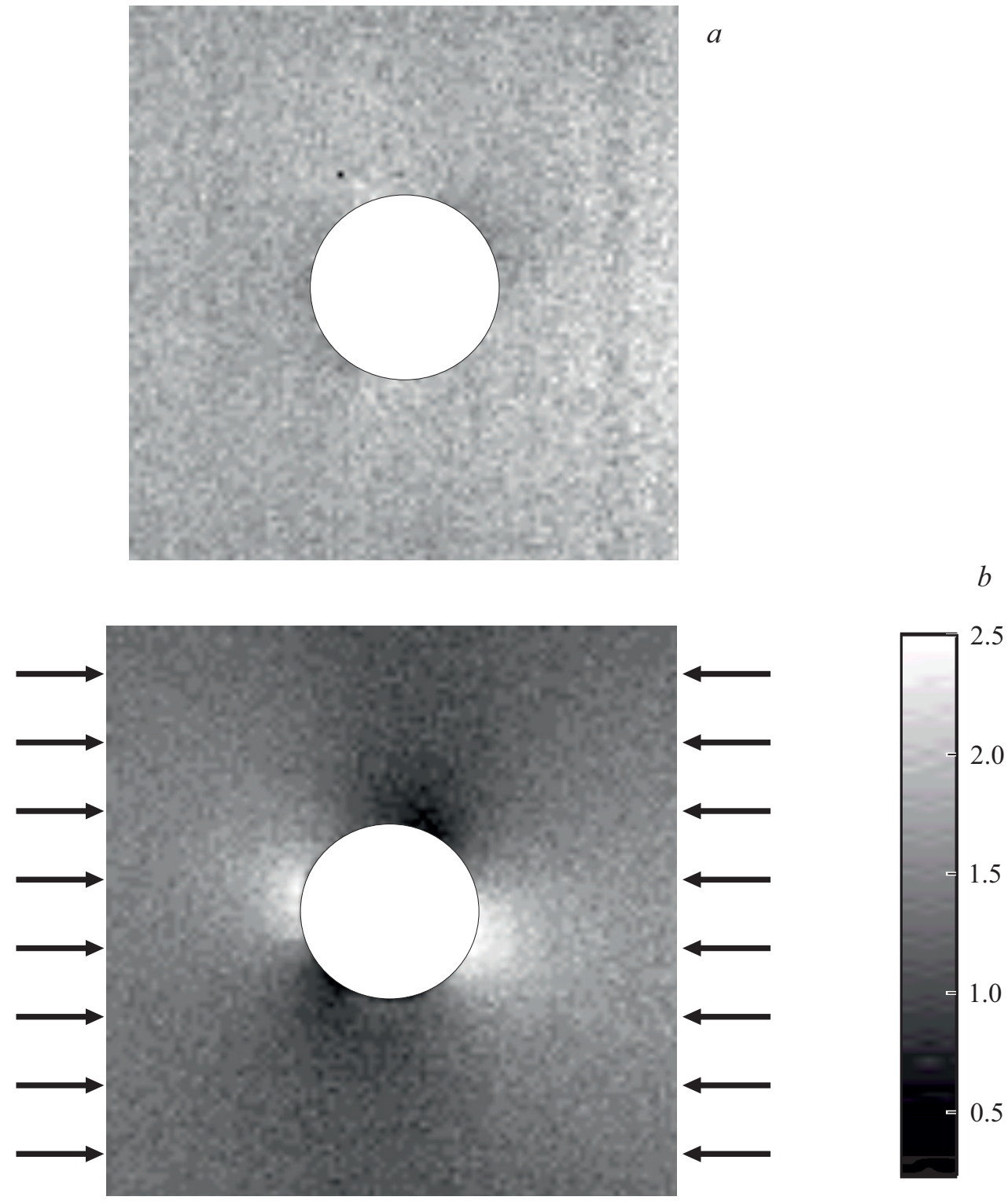

Оптоакустическое изображение области вокруг отверстия в пластине $\mathrm{Si}_{3} \mathrm{~N}_{4} . a-$ образец в исходном состоянии, $b-$ образец при сжимающем одноосном нагружении $27 \mathrm{MPa}$. Размер изображения $2.0 \times 2.0 \mathrm{~mm}$, частота модуляции $101 \mathrm{kHz}$. Приведена шкала интенсивности для оптоакустического сигнала в относительных единицах.

метрально симметрично вокруг отверстия. Одноосевое давление на боковые грани составило $27 \mathrm{MPa}$.

В выполненных в работе экспериментах длина тепловой волны в керамике была существенно меньше длины акустической волны, а длина последней значительно превышала размеры образца. В этом случае значение лазерного ультразвукового сигнала в соответствии с результатами работы [13] можно считать пропорциональным локальному значению коэффициента теплового расширения. В свою очередь, согласно решению задачи Кирша, в областях вблизи границы отверстия тензор напряжений при однородном сжатии или растяжении является одноосным. В соответствии с термодинамическими результатами $[7,8]$ в зонах действия одноосных напряжений зависимость коэффициента теплового расши- рения от напряжения является линейной и определяется соотношением

$$
\alpha=\alpha_{0}-\sigma E^{-2} \partial E / \partial T,
$$

где $\alpha_{0}$ - коэффициент линейного теплового расширения для недеформированного состояния, $E-$ модуль Юнга, $\sigma$ - одноосное напряжение. Таким образом, в соответствии с равенством (1) и результатами работы [13] для лазерных ультразвуковых сигналов в зонах действия одноосных напряжений должно соблюдаться равенство

$$
S=S_{0}(1+b \sigma),
$$

где $S_{0}$ - сигнал от образца в ненапряженном состоянии, $b$ - некоторый коэффициент пропорциональности, определяемый типом материала. 
Равенства (1) и (2) позволяют провести сравнение теоретических и экспериментальных результатов. Для керамики нитрида кремния $d E / d T=-0.012 \mathrm{GPa} / \mathrm{K}$, $\alpha_{0}=3.3 \cdot 10^{-6} \mathrm{~K}^{-1}$, среднее значение модуля Юнга $E=220 \mathrm{GPa}$, и, таким образом, в соответствии с термодинамическим результатом (1) в зонах действия одноосных напряжений должно выполняться соотношение

$$
\alpha=\alpha_{0}(1+0.8 \sigma[\mathrm{GPa}])\left[\mathrm{K}^{-1}\right]
$$

Из выражений (2), (3) и данных рисунка видно, что имеется качественное соответствие между экспериментальными и теоретическими результатами: лазерный ультразвуковой сигнал увеличивается в зонах действия растягивающих напряжений и уменьшается в зонах сжимающих напряжений. Однако количественный анализ показывает, что значение коэффициента $b$ должно быть существенно больше термодинамического и составлять примерно $12 \pm 2 \mathrm{GPa}^{-1}$. Это значение более чем на порядок превышает соответствующее значение коэффициента в формуле (3) для $\alpha$, которая учитывает только зависимость модуля упругости от температуры. Такое несоответствие свидетельствует о наличии дополнительного механизма влияния напряжений на коэффициент теплового расширения наряду с зависимостью модуля упругости от температуры.

В качестве такого механизма учтем образование дополнительных деформационных состояний в керамике при динамическом изменении ее температуры. В соответствии с подходом, разработанным в $[14,15]$, вероятность образования таких состояний в материале при наличии в нем напряжения $\sigma$ в единицу времени определяется выражением

$$
p=\frac{1}{\tau_{0}} \exp \left(-\frac{U-\Omega \sigma}{k_{\mathrm{B}} T}\right),
$$

где $\tau_{0}-$ время порядка периода колебаний атома в решетке, $U$ - энергия активации процесса дефектообразования, $\Omega-$ его активационный объем, $k_{\mathrm{B}}-$ постоянная Больцмана.

Согласно (4), для кинетики изменения концентрации возбужденных дефектных центров при изменении температуры можно записать уравнение

$$
\frac{\partial n}{\partial t}+\frac{n}{\tau}=\frac{n_{0}}{\tau_{0}} \exp \left(-\frac{U-\Omega \sigma}{k_{\mathrm{B}} T}\right)
$$

где $n_{0}-$ концентрация потенциальных дефектов в материале, возбуждаемых энергией активации $U ; \tau-$ время релаксации дефектного состояния.

Воспользовавшись подходом, описанным в работах $[6,16,17]$, можно показать, что возбуждение дефектных состояний за счет модуляции температуры образца в соответствии с законом (5) в линейном по температуре приближении сводится к введению некоторого эффективного коэффициента теплового расширения. В случае гармонического лазерного возбуждения с круговой частотой $\omega$, удовлетворяющей условию $\omega \tau \ll 1$, и при $\Omega \sigma\left(k_{\mathrm{B}} T_{0}\right)^{-1}<1$ его значение определяется соотношением

$$
\alpha_{e f f} \cong \alpha_{0}+\frac{n_{0} \tau}{\tau_{0}} \frac{\Omega^{2} \sigma}{k_{\mathrm{B}} T_{0}^{2}} \exp \left(-\frac{U}{k_{\mathrm{B}} T_{0}}\right)=\alpha_{0}(1+b \sigma),
$$

где

$$
b=\frac{n_{0} \tau}{\alpha_{0} \tau_{0}} \frac{\Omega^{2}}{k_{\mathrm{B}} T_{0}^{2}} \exp \left(-\frac{U}{k_{\mathrm{B}} T_{0}}\right),
$$

$T_{0}$ - температура окружающей среды.

Выражение (6) позволяет оценить величину коэффициента $b$ при типичных значениях входящих в него параметров. Будем считать, что $n_{0} \cong 10^{20} \mathrm{~cm}^{-3}$, $\Omega \cong 10^{-28} \mathrm{~m}^{-3}, \alpha_{0}=3.3 \cdot 10^{-6} \mathrm{~K}^{-1}, \tau \cong 10 \tau_{0}$. Тогда для оценки экспоненты, входящей в (6), необходимо знать значение энергии активации. Для объемных областей решетки керамики нитрида кремния ее значение достаточно велико, но из-за присутствия границ зерен, полостей и дефектов по данным работы [18] оно может быть значительно меньше и составлять только $15.46 \mathrm{~kJ} \cdot \mathrm{mol}^{-1}$, что соответствует около $0.16 \mathrm{eV}$. При такой энергии активации значение экспоненты при комнатной температуре находится на уровне $10^{-3}$. В этом случае произведение $b \sigma$ может сравниваться с единицей при значениях напряжений на уровне $10 \mathrm{MPa}$, что приблизительно соответствует полученным экспериментальным данным.

В заключение следует отметить, что в соответствии с проведенным рассмотрением при лазерной генерации ультразвука по термоупругому механизму в керамике нитрида кремния при наличии механических напряжений заметный вклад в сигнал может быть связан с присутствием в ней дефектных состояний.

\section{Конфликт интересов}

Авторы заявляют, что у них нет конфликта интересов.

\section{Список литературы}

[1] A. Setiawan, G.B. Suparta, M. Mitrayama, W. Nugroho, Int. J. Adv. Sci. Eng. Inform. Technol., 7 (6), 2189 (2017). DOI: $10.18517 /$ ijaseit.7.6.2816

[2] D.D. Markushev, J. Ordonez-Miranda, M.D. Rabasović, M. Chirtoc, D.M. Todorović, S.E Bialkowski, D. Korte, M. Franko, Eur. Phys. J. Plus., 132 (1), 33 (2017). DOI: $10.1140 /$ epjp/i2017-11307-2

[3] C. Millon, A. Vanhoye, A.F. Obaton, J.D. Penot, Welding World, 62 (3), 653 (2018). DOI: 10.1007/s40194-018-0567-9

[4] Yu.V. Sudenkov, B.A. Zimin, Int. J. Heat Mass Transfer, 85, 781 (2015). DOI: 10.1016/j.ijheatmasstransfer.2015.01.119

[5] Yu.V. Sudenkov, B.A. Zimin, V.E. Sventitskaya, AIP Conf. Proc., 1959, 070035 (2018). DOI: 10.1063/1.5034710

[6] A.L. Glazov, K.L. Muratikov, J. Appl. Phys., 128 (9), 095106 (2020). DOI: $10.1063 / 5.0013308$

[7] A.K. Wong, R. Jones, J.G. Sparrow, J. Phys. Chem. Solids, 48 (8), 749 (1987). DOI: 10.1016/0022-3697(87)90071-0 
[8] R.J. Greene, E.A. Patterson, R.E. Rowlands, in: Springer handbook of experimental solid mechanics, ed by W. Sharpe (Springer, Boston, 2008), p. 743.

DOI: 10.1007/978-0-387-30877-7_26

[9] K.L. Muratikov, A.L. Glazov, D.N. Rose, J.E. Dumar, J. Appl. Phys., 88 (5), 2948 (2000). DOI: 10.1063/1.1287526

[10] К.Л. Муратиков, А.Л. Глазов, Д.Н. Роуз, Д.Е. Думар, Письма в ЖТФ, 28 (9), 48 (2002).

[11] К.Л. Муратиков, А.Л. Глазов, ЖТФ, 73 (8), 90 (2003).

[12] S. Timoshenko, J.N. Goodier, Theory of elasticity (McGrawHill, N.Y., 1951), p. 78.

[13] R.G. Stearns, G.S. Kino, Appl. Phys. Lett., 47 (10), 1048 (1985). DOI: 10.1063/1.96374

[14] S.N. Zhurkov, Int. J. Fract. Mech., 1 (4), 311 (1965). DOI: $10.1007 / \mathrm{BF} 03545562$

[15] S.N. Zhurkov, V.E. Korsukov, J. Polym. Sci.: Polym. Phys. Ed., 12 (2) 385 (1974). DOI: 10.1002/pol.1974.180120211

[16] А.Л. Глазов, К.Л. Муратиков, Письма в ЖТФ, 45 (17), 51 (2019). DOI: 10.21883/PJTF.2019.17.48226.17892

[17] А.Л. Глазов, К.Л. Муратиков, Письма в ЖТФ, 46 (10), 18 (2020). DOI: 10.21883/PJTF.2020.10.49425.18247

[18] S. Shao, H. Luo, L. Deng, J. He, S. Huang, AIP Adv., 8 (7), 075127 (2018). DOI: 10.1063/1.5033965 